\title{
Sliding mechanisms in tribological contact of TMD-C sputtered coatings
}

\author{
T. Polcar ${ }^{1,2} \&$ A. Cavaleiro ${ }^{2}$ \\ ${ }^{1}$ Department of Control Engineering, Faculty of Electrical Engineering, \\ Czech Technical University in Prague, Czech Republic \\ ${ }^{2}$ SEG-CEMUC, Mechanical Engineering Department, \\ University of Coimbra, Portugal
}

\begin{abstract}
In this paper, results on the tribological behaviour of nanocomposite coatings composed by nanoplatelets of transition metal dichalcogenides (TMD) immersed in a C-rich amorphous matrix will be presented. It will be shown that this microstructure will allow low friction coefficients in wide range of surrounding environments, since TMD platelets are re-oriented in the C-matrix as a consequence of the mechanical energy liberated in the sliding contact. In this case, the oxidation of the TMD material is avoided due to the protection achieved by the $\mathrm{C}$ matrix. Examples of TMD-C coatings of the W-S-C and Mo$\mathrm{Se}-\mathrm{C}$ systems will be presented and discussed. After tribological characterization of the coatings in different environments under different loading conditions experimental evidence of the wear mechanisms will be given by cross section transmission electron microscopy, Raman spectroscopy and Auger electron spectroscopy.
\end{abstract}

Keywords: sliding mechanisms, self-lubrication coatings, low friction, TMD coatings.

\section{Introduction}

The idea behind the use of composite coatings, including different well-known phase materials with good tribological performance, was to achieve a synergetic effect that can overcome the individual drawbacks of each specific phase in low friction demands. One of the most interesting materials for the nanocomposite design of low friction coatings is any member of the transition metal 
dichalcogenides (TMD) family. The weak Van der Waals forces between the lamellae of TMD allow an almost frictionless sliding, providing that it is performed in an absolutely clean environment, preferentially ultra-high vacuum [1]. Unfortunately, TMD is very vulnerable to environmental attacks; the presence of oxygen and water vapour hinders its excellent frictional properties.

Pulsed laser deposition and magnetron sputtering have been the most studied ways to prepare thin TMD films; the latter method is widely used in industrial scale for TMD coatings preparation [2]. Pure magnetron sputtered TMD films perform well in vacuum conditions, inert atmospheres or, to a less extent, in dry air. Nevertheless, even for such restricted application conditions, they suffer from a lack of adhesion to the steel substrates, low resistance to abrasive wear due to their low hardness and, generally, low load-bearing capacity. It should be pointed out that the optimum orientation of the TMD phase assuring a minimum friction, (002), is almost impossible to achieve except for very thin films $[1,3]$. Therefore, a complicate process is required to achieve the low friction; in general, the TMD phase must be "reoriented", either by removal of the film material and consequent formation of a tribolayer or by reorientation of the TMD phases inside the carbon matrix.

On the other hand, the environmental attacks are difficultly overcome due to another typical feature of magnetron sputtered TMD films, their columnar structure, which allows the penetration of the oxygen or the water vapour inside the coatings $[4,5]$. Finally, the adhesion and cohesion of such films is limited despite the improvement achieved with the use of metallic interlayers (mostly titanium).

To face the issues referred to above, several concepts were envisaged. One of the most successful solutions was alloying TMD with an additional metallic (Ti $[2,6], \mathrm{Pb}[7], \mathrm{Au}[8])$ or non-metallic $(\mathrm{C}[9,10], \mathrm{N}[11])$ elements. The main feature of the alloyed films was the improvement of the microstructure density, the enhancement of the hardness and, therefore, the increase in the load-bearing capacity.

In this paper, a new concept of TMD films co-sputtered with carbon based on the tailoring of the microstructure, which leads to excellent tribological properties, is presented.

\section{New coatings design}

In a first set of studies, W-S-C films were co-deposited by sputtering from a $\mathrm{WS}_{2}$ target in a $\mathrm{CH}_{4}$-containing reactive atmosphere. The flow of $\mathrm{CH}_{4}$ and the target power were varied to prepare a large range of chemical compositions from pure $\mathrm{WS}_{2}$ to W-S-C with carbon contents up to 70 at.\%; the S/W ratio was about 1.2 . Fig. 1(a) clearly shows that the main disadvantage of TMD films, the detrimental effect of the humid air, was not overcome and the friction in these conditions was relatively high whatever the carbon content was $[9,11]$. However, the rapid film failure typical of pure tungsten disulphide was not observed and the coating wear rate was quite low (Fig. 1(b)), result attributed to the significant increase of the hardness, from $0.5 \mathrm{GPa}\left(\mathrm{WS}_{2}\right)$ to $\sim 10 \mathrm{GPa}$, achieved with the $\mathrm{C}$ incorporation. 


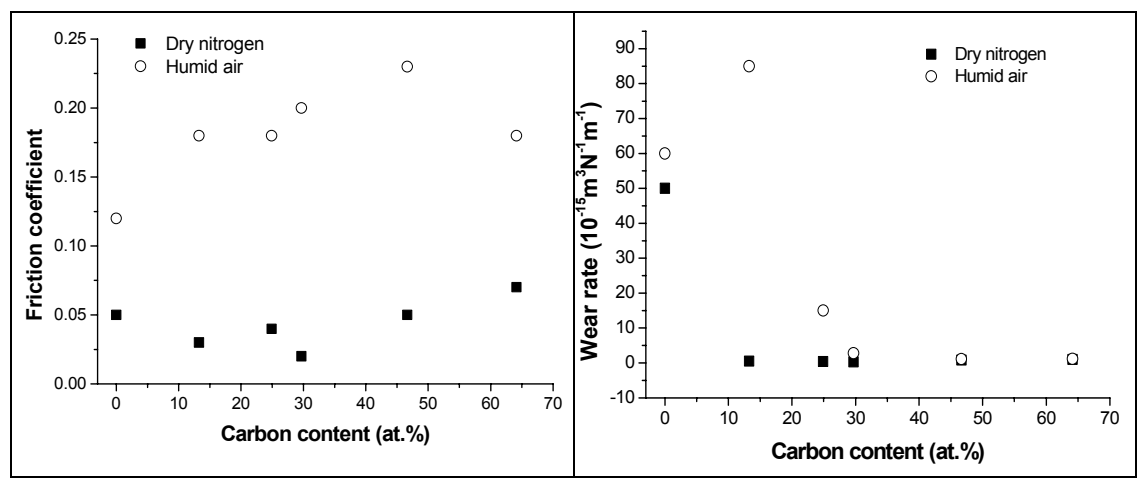

(a)

Figure 1: Friction, (a), and wear, (b), coefficients of $\mathrm{W}-\mathrm{S}-\mathrm{C}$ reactive deposited sputtered coatings as a function of the $\mathrm{C}$ content when tested in humid air and dry $\mathrm{N}_{2}(\operatorname{load} 5 \mathrm{~N})$.

The analysis of the microstructure of the reactive deposited W-S-C coatings [5] allowed one to conclude that, for high carbon content, they were formed by a mixture of nanocrystals of $\mathrm{WS}_{2}$ phase side by side with nanocrystals of W-C phases and small C-based amorphous zones (see example in Fig. 2), or just by $\mathrm{WS}_{2}$ nanograins dispersed in the amorphous carbon matrix (low carbon content). A similar behaviour was observed elsewhere for $\mathrm{WS}_{2} / \mathrm{DLC} / \mathrm{WC}$ coatings showing a huge friction coefficient difference when sliding in humid or dry air [10]; the friction behaviour was attributed to the alternative formation of a $\mathrm{WS}_{2}$ rich or a $\mathrm{C}$-rich layer during the cyclic change of the environment from dry to humid, respectively. Another concept presented in literature for the TMD $+\mathrm{C}$ composite coatings deals with nanolayered structured coatings [12], where tungsten disulphide and carbon layers form a "super-lattice" film (the thickness of layers is in nanometre scale). The performance of such films was only moderate.

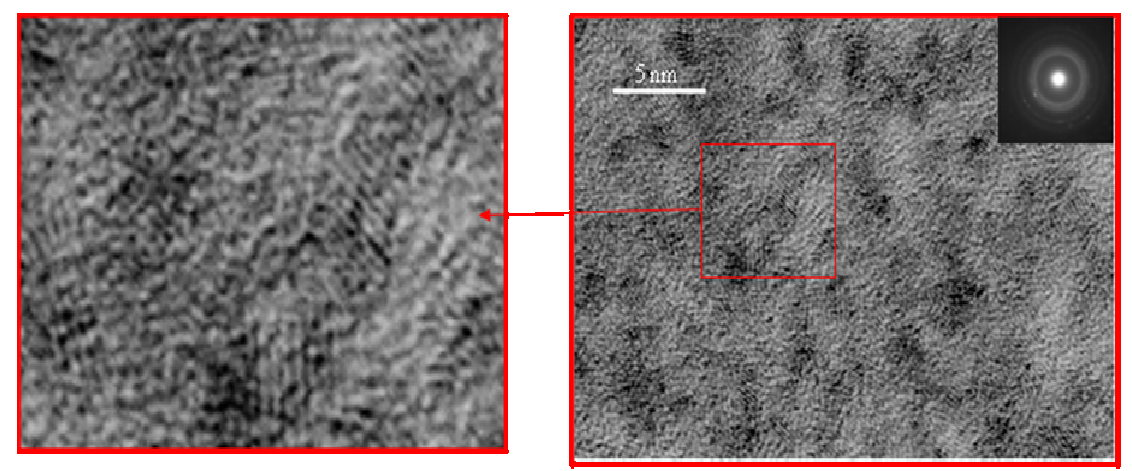

Figure 2: $\quad$ TEM micrographs of the reactive sputtered $\mathrm{W}_{17} \mathrm{~S}_{15} \mathrm{C}_{68}$. 
All these three different coating microstructures schematically shown in Fig. 3 proved to be unsatisfactory for reducing the friction coefficient in humid air sliding conditions. The multilayer films are vulnerable to cohesion damage, since interlayers sliding can occur in the bulk of the film where the shear stresses are larger than at the surface. This problem can be partially solved by thinning of layers down to units of nanometres; however, such a superlattice structure is difficult to be achieved on typical industrial substrates with standard surface roughness.
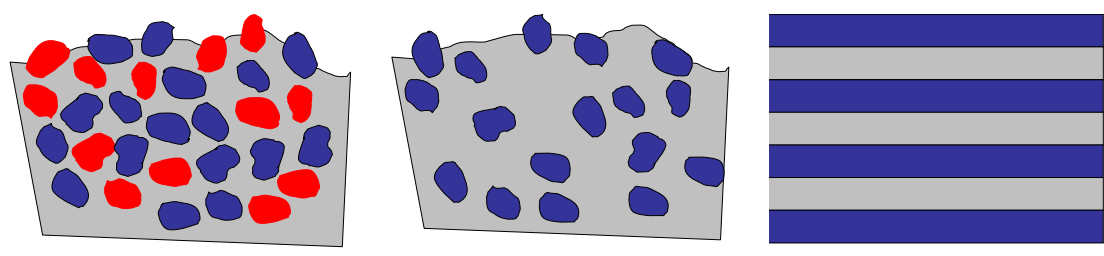

Figure 3: Schematic view of previous W-S-C films designs: Nanocomposite coatings with hard WC nanoparticles embedded together with $\mathrm{WS}_{2}$ nanonograins in an amorphous carbon matrix (left); nanograins of $\mathrm{WS}_{2}$ in a C-matrix (center); super-lattice $\mathrm{WS}_{2}+\mathrm{C}$ layers film.

On the other hand, the other two microstructures cannot guarantee simultaneously that either the TMD nanocrystals could be re-oriented with the basal planes parallel to the sliding distance or the oxidation of the dangling bonds should not occur. In fact, during the re-orientation process the extremities of the grains will enter directly in contact with the atmosphere being immediately oxidized.

\section{Approach}

The basis for the achievement of a low friction coating in a great variety of environments could be envisaged by a micro(nano)structure where TMD nanocrystals could be deposited completely enrobed in a protective C-rich matrix. If possible, these nanocrystals should have shapes that allow an easy reorientation of the basal planes parallel to the sliding direction. C-matrix will contribute for improving the hardness and the compactness of the coatings, as well as for protecting the TMD against the oxidation until the moment that they are completely aligned and they arise to the contact surface. By their side, TMD should assure the low friction coefficients, due to the easy sliding of the basal planes, and only a few amounts of strong bonds, promoted by the oxygen, should exist. In parallel with this approach, the use of other types of TMDs with less sensibility to the oxidation, such as $\mathrm{MoSe}_{2}$ [13], should also be envisaged.

During sliding in pin-on-disk testing, strong shear stresses occur which could contribute for the re-orientation of the TMD crystals in the C-based amorphous matrix. However, during the alignment of the TMD nanocrystals parallel to the sliding direction, their contact with the atmosphere gives rise to the reaction of 
the dangling bonds of the end plans with oxygen with the consequent increase of the friction. In pure TMD coatings this phenomenon was not observed due to the porous structure and very low hardness giving rise to very high wear rate [3]. Therefore, as generally accepted in the wear models it is expected that TMD material is worn out and then adheres on both surfaces in the contact. Such tribolayer is very thin (nanometre scale) with highly oriented TMD phase, mainly with the basal plans parallel to film surface $[1,3]$.

The problems referred to above could be avoided if the crystals were already aligned in the material, since in this case almost no dangling bonds would be available for reaction [14]. Recently, it was possible to prove that nanocrystals of a compound, such as TiC, could be re-arranged through the C-matrix where they were embedded, during the mechanical deformation of the material by indentation [15]. It could be possible that TMD-C films, where nanograins of TMD are embedded in an amorphous carbon matrix, exhibit similar behaviour. Nevertheless, the re-orientation of TMD crystals in a carbon matrix has not been studied yet.

Thus, it was decided to prepare TMD-C films with very small nanograins of the TMD phase (reduced, if possible, to one cell of TMD), since small grain size could promote more easily the re-orientation of the TMD phase inside the carbon matrix.

\section{How to deposit the coatings with this nanocomposite microstructure?}

In order to deposit coatings containing simultaneously $\mathrm{C}$ and the TMD phase with a new microstructure, the deposition by co-sputtering from TMD and $\mathrm{C}$ sources, either from separate targets or by a puzzled target with both materials (Fig. 4), can be an alternative.
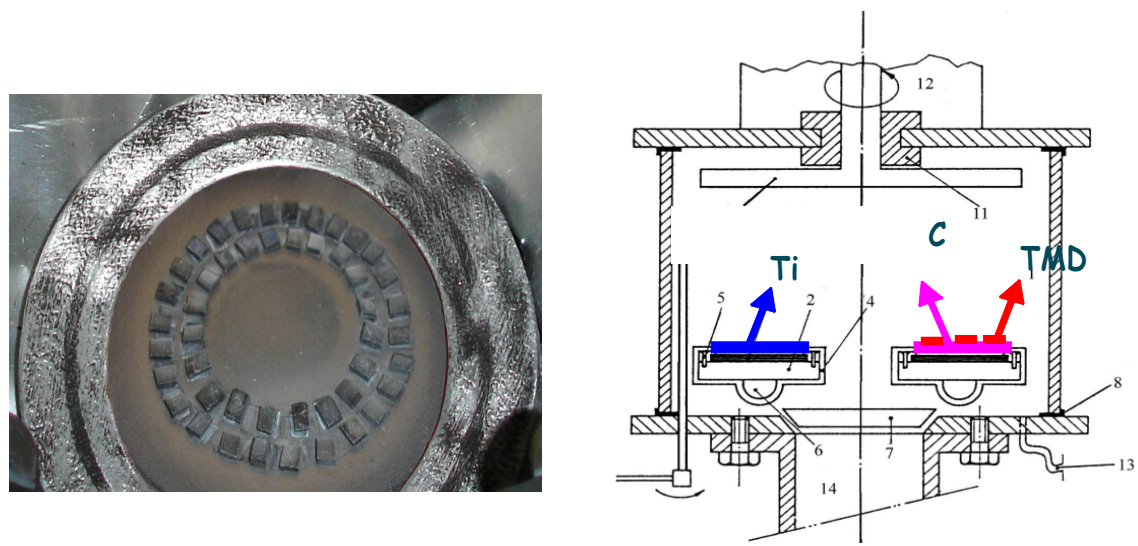

Figure 4: Schema of the deposition chamber with two electrodes and picture of the puzzled target. For separate target deposition, the Ti target is replaced by a TMD one whereas in the other electrode only a $\mathrm{C}$ target is attached. 

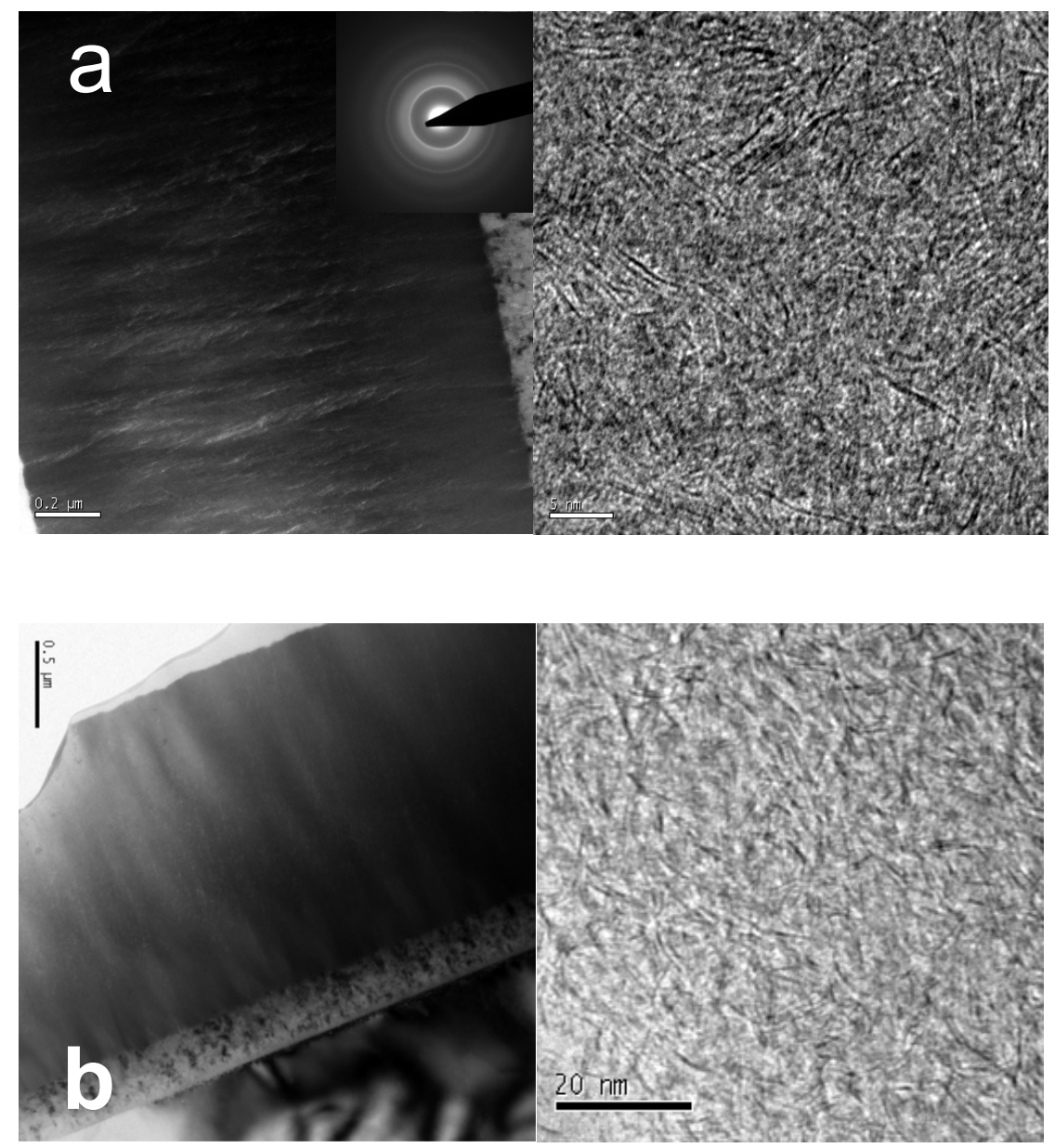

Figure 5: TEM and HR-TEM images of W-S-C (a) and Mo-Se-C (b) cosputtered films deposited with $\sim 50$ at.\% C. Note the dense structure (left) and the separated TMD platelets (right).

With this geometry, microstructures such as those shown in Fig. 5 for Mo-Se-C and $\mathrm{W}-\mathrm{S}-\mathrm{C}$ films with $\sim 50$ at. $\% \mathrm{C}$ can be deposited $[16,17]$. The TMD phase is under a platelet shape with only a couple of stacked basal planes. The platelets are completely enrobed by the C-matrix. X-ray diffraction and Raman spectroscopy undoubtedly confirmed the presence of the TMD and the $\mathrm{C}$ phases [18-20]. The hardness was about 3 and $10 \mathrm{GPa}$ for Mo-Se-C and W-S-C, respectively, compared to the $<0.5 \mathrm{GPa}$ of the pure TMD films sputtered under identical conditions from the TMD target. 


\section{Friction performance}

Figures 6 a) and b) show the friction coefficient obtained in a sliding reciprocating pin-on-disk test (frequency - $20 \mathrm{~Hz}$; stroke length - $1 \mathrm{~mm}$ ) for two coatings of the systems W-S-C and Mo-Se-C with approximately 50 at.\% C. Two facts are evident: (i) in humid conditions Mo-Se-C coatings show better friction performance than W-S-C ones; (ii) in some loading conditions (very high applied loads) the friction coefficient in humid air approaches that in dry conditions. This last result gives strong evidence that the scope of re-orientation of the TMD nanocrystals, in conditions of oxidation protection, can be achieved. However, important amounts of mechanical energy have to be used to allow the correct reorientation in the envisaged conditions. The progressive decrease of the friction coefficient with increasing applied load suggests that the sliding process is driven by the TMD phases in the contact (such decrease is typical of TMD [21]), since pure carbon films identical to the amorphous carbon matrix of these coatings exhibit a slight increase of the friction with load [16]. It is also important to remark that a similar behavior is found in dry conditions although with a starting value much lower $(\sim 0.08$ against $\sim 0.20$ for a $20 \mathrm{~N}$ load$)$.

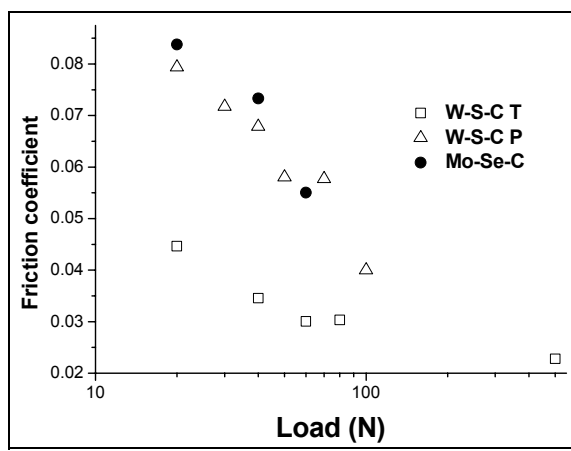

(a)

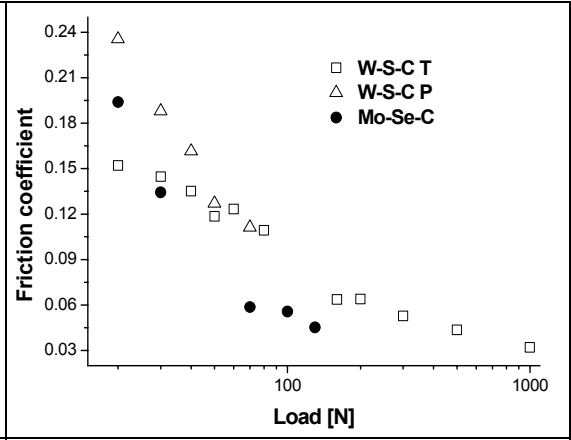

(b)

Figure 6: Friction coefficient of TMD-C coatings as a function of the applied load in (a) dry and (b) humid conditions. Note logarithmic load scale.

Figure 7 shows the average friction coefficient obtained in tests with increasing duration times when a very low load $(5 \mathrm{~N})$ is applied to the ball. As can be confirmed, there is a steep decrease of the friction coefficient in the first thousands of cycles being possible to reach a steady state value $(\sim 0.05)$ close to 100,000 cycles, which is not far from the lowest value showed in figure 6 for these coatings when tested with much higher loads. In another paper of the authors, it was shown the evolution of the friction coefficient as a function of the number of cycles for a W-S-C coating tested by changing the humidity in the atmosphere every 10,000 cycles [22]. Besides the progressive approach between the friction values in both, dry and humid, environments with increasing test duration, also in both cases a decreasing trend in each step could be observed. 


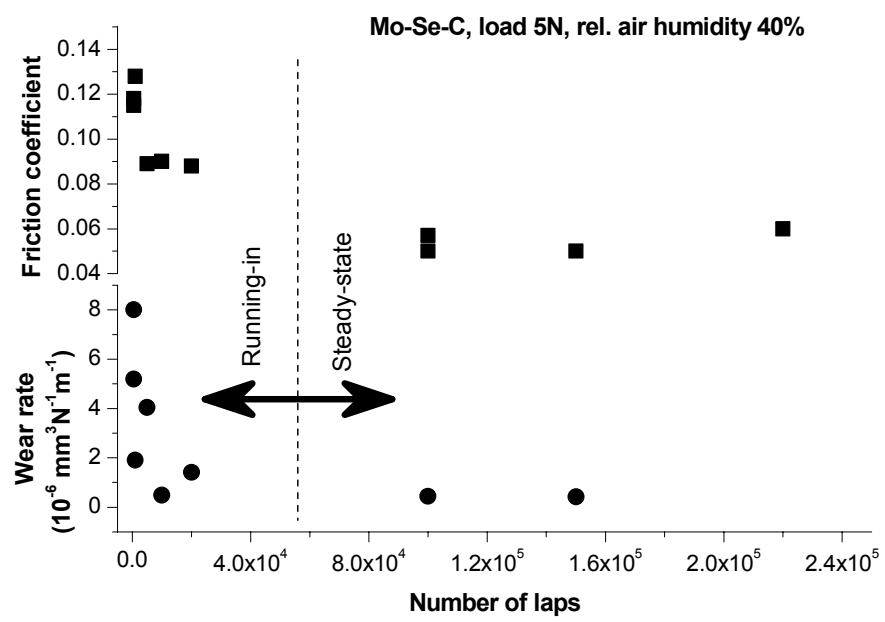

Figure 7: Evolution of the friction coefficient and the wear rate after tests with different duration of a Mo-Se-C coating sliding under room conditions with an applied load of $5 \mathrm{~N}$ (pin-on-disc, 100Cr6 ball with a diameter of $6 \mathrm{~mm}$ ). The steady state wear regime was reached after long running-in.

In conclusion, in sliding tests of TMD-C coatings, low friction coefficients can be achieved in any environment provided that sufficient contact pressure or test duration are used.

\section{Evidence of the sliding mechanism}

The progressive way as to how the formation of the TMD tribolayer occurs can be demonstrated experimentally by the analysis of the top surface of the sliding track after wear testing. Figure 8 shows the Raman spectra of TMD-C films obtained after tribological tests carried out with either the same number of cycles but different loads or the same load but different number of cycles. It is clear that the increase in the load and/or the number of cycles gives rise to a more intense signal of the bands assigned to the TMD phase in comparison to those of the Cbased material ( $\mathrm{G}$ and $\mathrm{D}$ bands). This result confirms an increasing agglomeration of TMD material close to the contact zone.

In previous studies $[16,17]$, Auger spectroscopy (AES) showed that the TMD signal increase in Mo-Se-C coatings was due to the thickening of the surface TMD tribolayer when contact pressure and/or number of laps (i.e. test duration) was increased. It was clear the absence of the $\mathrm{C}$-signal on the top layer suggesting that this was formed almost exclusively by TMD. However, the agglomeration of the TMD material in the contact zone should only lead to the reduction of the friction coefficient providing that its basal planes are aligned parallel to the surface and no abundant oxidation occurs that can lead to strong bonding between them. It is therefore also important to remark the absence of $\mathrm{O}$ in this top layer (compare to the residual signal of the material underneath) 


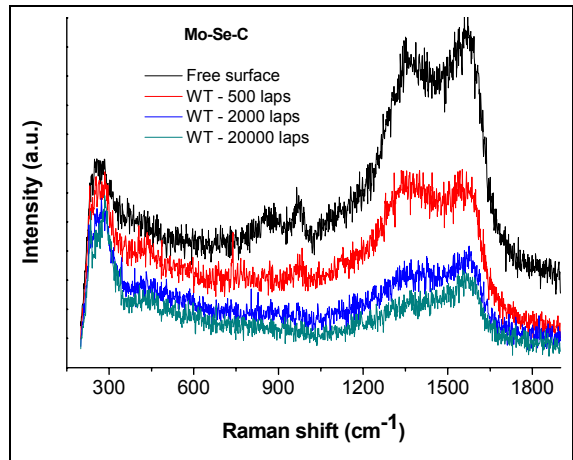

(a)

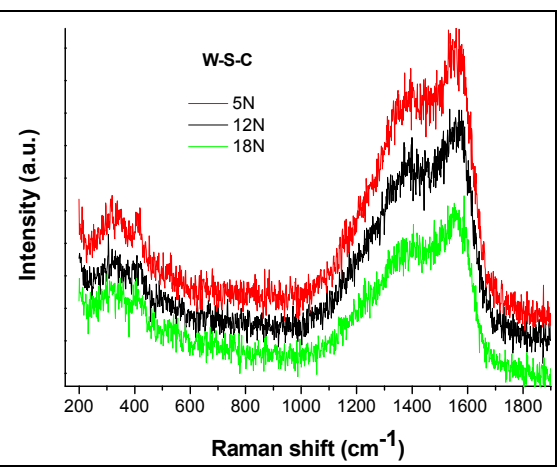

(b)

Figure 8: Raman spectra taken from the center of the wear track as a function of the test duration (a) and applied load (b).
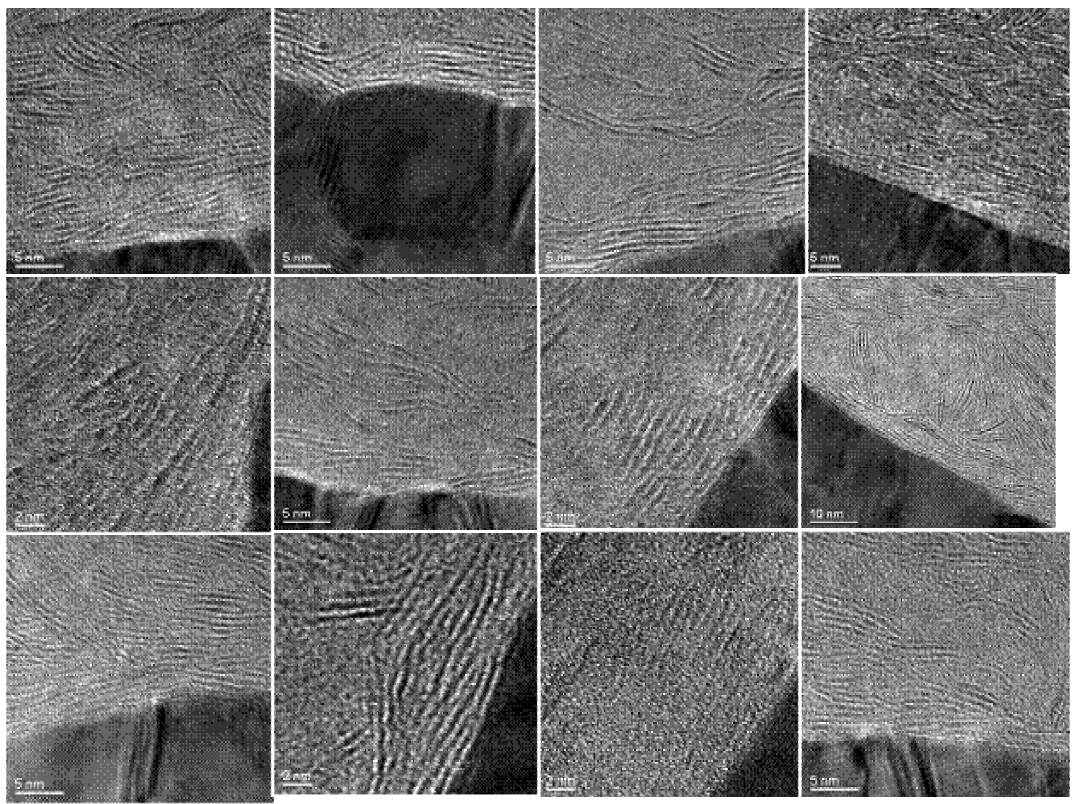

Figure 9: HR-TEM images of the cross-section of the wear tracks prepared by Focused Ion Beam. The black in the images is the protective gold layer sputtered over the wear track. The preferential orientation of the TMD platelets is clearly visible contrasting to asdeposited state shown in Fig. 5.

stating that the formation of the tribolayer was achieved in a protective way, without the oxidation of the dangling bonds of the original short TMD platelets. 
The microstructural observation of the cross section of the top layer of the wear track by HRTEM (high-resolution transmission electron microscopy) showed that the TMD material was aligned parallel to the surface covering the most part of the zones which were analyzed, see Fig. 9. Furthermore, the same micrograph shows that as the analysis is being moved to the interior of the film, the alignment is less and less evident. The observation of a great number of micrographs of different zones of the wear track [17] permitted concluding that the reorientation of the TMD platelets is made progressively from some tens of nanometers until the track surface. Almost no other orientations but parallel to the surface are encountered in the first $5 \mathrm{~nm}$ top layer.

\section{Conclusions}

The deposition of nanocomposite coatings of the W-S-C and Mo-Se-C systems by co-sputtering from $\mathrm{WS}_{2} / \mathrm{MoSe}_{2}$ and $\mathrm{C}$ materials originated a nanocomposite structure composed by $\mathrm{WS}_{2} / \mathrm{MoSe}_{2}$ platelets embedded in a C-rich amorphous matrix. With this morphology, during the tribological testing the nano-platelets could be re-oriented parallel to the sliding direction giving rise to low friction coefficients. It was demonstrated that the C-matrix had two roles: (i) it contributed to the increase of the hardness and load bearing capacity and (ii) it avoided the oxidation of the TMD platelets during re-orientation permitting low friction coefficients in O-containing environments. In order to achieve a correct reorientation of the TMD platelets, enough mechanical energy was required, explaining why the friction coefficient progressively decreased when the duration of the test increased, at the same applied load, or the load increased (for constant test durations). The experimental evidence of the friction performance was given by high resolution transmission electron microscopy, Raman spectroscopy and Auger spectrometry.

\section{Acknowledgements}

This work was supported by the Czech Science Foundation through the project 106/07/P014, by the Ministry of Education of the Czech Republic (project MSM 6840770038), by EU through the FOREMOST project and by the Fundação para a Ciência e a Tecnologia through the project SFRH/BPD/34515/2006.

\section{References}

[1] Lansdown, A.R., Molybdenum Disulphide Lubrication, Elsevier: Amsterdam, 1999.

[2] Renevier, N.M., Fox, V.C., Teer, D.G., Hampshire, J., Coating characteristics and tribological properties of sputter-deposited MoS2/metal composite coatings deposited by closed field unbalanced magnetron sputter ion plating Surface Coatings Technology, 127(1), pp. 24-37, 2000. 
[3] Moser, J., Lèvy, F., MoS2-x lubricating films - structure and wear mechanisms investigated by cross-sectional transmission electronmicroscopy. Thin Solid Films, 228(1-2), pp. 257 -260, 1993.

[4] Regula, M., Ballif, C., Moser, J.H., Lévy, F., Structural, chemical, and electrical characterisation of reactively sputtered WSx thin films. Thin Solid Films, 280(1-2), pp. 67-75, 1996.

[5] Nossa, A., Cavaleiro, A., Chemical and physical characterization of $\mathrm{C}(\mathrm{N})$ doped W-S sputtered films. Journal of Materials Research, 19(8), pp. 2356 $-2365,2004$.

[6] Teer, D.G., New solid lubricant coatings. Wear, 251, pp. 1068-1074, 2001.

[7] Wahl, K.J., Seitzman, L.E., Bolster, R.N., Singer, I.L., Low-friction, highendurance, ion-beam-deposited $\mathrm{Pb}-\mathrm{Mo}-\mathrm{S}$ coatings. Surface Coatings Technology, 73(3), 152-159, 1995.

[8] Simmonds, M.C., Savan, A., Pfluger, E., Van Swygenhoven, H., Mechanical and tribological performance of MoS2 co-sputtered composites. Surface Coatings Technology 126(1), pp. 15-24, 2000.

[9] Evaristo, M., Nossa, A., Cavaleiro, A., W-S-C sputtered films: Influence of the carbon alloying method on the mechanical properties Surface Coatings Technology, 200(1-4), pp. 1076-1079, 2005.

[10] Voevodin, A.A., O'Neill, J.P., Zabinski, J.S., Nanocomposite tribological coatings for aerospace applications. Surface Coatings Technology, 116, pp. 36-45, 1999.

[11] Nossa, A., Cavaleiro, A., Tribological behaviour of N(C)-Alloyed W-S films. Tribology Letters, 28(1), pp. 59-70, 2007.

[12] Noshiro, J., Watanabe, S., Sakurai, T., Miyake, S., Friction properties of co-sputtered sulfide/DLC solid lubricating films. Surface Coatings Technology, 200(20-21), pp. 5849-5854, 2006.

[13] Kubart, T., Polcar, T., Kopecký, L., Novák, R., Nováková, D., Temperature dependence of tribological properties of MoS2 and MoSe2 coatings. Surface Coatings Technology, 193(1-3), pp. 230-233, 2005.

[14] Evaristo, M., Polcar, T., Cavaleiro, A., Tribological behaviour of C-alloyed transition metal dichalcogenides (TMD) coatings in different environments. International Journal of Mechanics and Materials in Design, 4, pp. 137-143, 2008.

[15] Chen, C.Q., Pei, Y.T., Shaka, K.P., De Hosson, J.M.T., Nanoscale deformation in TiC/a-C multilayered nanocomposite coatings. Applied Physics Letters, 92(24), 241913, 2008.

[16] T. Polcar, M. Evaristo and A. Cavaleiro, Can W-Se-C coatings be competitive to W-S-C ones?. Plasma Processes \& Polymers, 6,DOI: 10.1002/ppap.200930414

[17] Polcar, T., Evaristo, M., Colaço, R., Sandu, C. Silviu, Cavaleiro, A., Nanoscale triboactivity: The response of Mo-Se-C coatings to sliding. Acta Materialia, 56(18), pp. 5101-5111, 2008.

[18] Polcar, T., Evaristo, M., Cavaleiro, A., The tribological behavior of W-S-C films in pin-on-disk testing at elevated temperature. Vacuum, 81(11-12), pp. 1439-1442, 2007. 
[19] Polcar, T., Evaristo, M., Cavaleiro, A., Friction of self-lubricating W-S-C sputtered coatings sliding under increasing load. Plasma Processes \& Polymers, 4, pp. S541-S546, 2007.

[20] Polcar, T., Evaristo, M., Stueber, M., Cavaleiro, A., Synthesis and structural properties of Mo-Se-C sputtered coatings. Surface Coatings Technology, 202(11), pp. 2418-2442, 2008.

[21] Singer, I.L., Bolster R.N., Wegand, J., Fayeulle, S., Stupp, B.C., Hertzian stress contribution to low friction behavior of thin $\mathrm{MoS}_{2}$ coatings. Applied Physics Letters, 57(10), pp. 995-997, 1990.

[22] Polcar, T., Evaristo, M., Cavaleiro, A., Comparative study of the tribological behavior of self-lubricating W-S-C and Mo-Se-C sputtered coatings. Wear, 266(3-4), pp. 388-392, 2009. 